\title{
Energy Efficient MIH based Independent and Heterogeneous Vertical Handover Algorithm
}

\author{
S. S. Sambare \\ Department of Computer \\ Engineering, Pimpri Chinchwad \\ College of Engineering Pune-44
}

\author{
M. U. Kharat, $\mathrm{PhD}$ \\ Department of Computer \\ Engineering, MET, Nashik
}

\author{
Avinash U. Jadhav \\ Department of Computer \\ Engineering, Pimpri Chinchwad \\ College of Engineering Pune-44
}

\begin{abstract}
In recent years wireless technologies are most popular in modern world; similarly in communication field also essential for multi-mode wireless devices in heterogeneous wireless network environments. To make a seamless handover among heterogeneous networks, the optimal selection of a network is a challenging task. The main issue in RAT is to manage the handover efficiency between one region to other region communication. Handover is the process of communicating from one region to other region without any interruptions; we apply Media Independent Handover (MIH) method with global access point services. Through this we can show the communication can be carried out by the Access point associated with Base station, so that the Source can efficiently communicate with any mobile users (nodes) presented in any regions with full independent support. So that the handover based communication is achieved via MIH methodology. Quality of Service (QOS) is the main concern over wireless communications, so that the successful route establishments will be achieved via Route Request and Route Response methodologies. Source and destinations are communicated via route requesting and response methodology, if any neighbor node fails to send response back to request that will be considered as the Malicious or abnormal node, as well as I will not be a part for further precedence in communications. In simulation environment, apart from the paper implementations we can show multicasting routing methodology to show our results are best with more destinations as well. For all the proposed results should prove the energy efficiency is more, delay is reduced, performance and network lifetime is improved, we show these details via graphical results.
\end{abstract}

\section{Keywords}

Energy efficiency, vertical handover, handover decision algorithm, Energy efficiency

\section{INTRODUCTION}

Mobile communication has become more widespread due to increased availability of portable devices and advanced wireless technology. Moreover, the core network of heterogeneous wireless access networks, e.g. WLAN [1], WiMAX [2,3], and 3GPP (WCDMA, LTE); they are evolving into all-IP based network.

In the first step, the mobile terminal (MT)discovers its available neighboring networks. During the handoff decision phase, mobile device decides whether the connection to be continued with current network or to be switched over to another one. The decision may depend on various parameters including the available bandwidth, delay, jitter, bit error rate, cost, transmit power, current battery status of the mobile device, and the user's preferences. During the handoff execution phase, connections need to be re-routed from the existing network to the new network in a seamless manner. Due to integration of various wireless technologies, terminal power consumption and handoff

\subsection{Media Independent Handover (MIH)}

The Media-Independent Handover (MIH) standard is part of the IEEE 802.21 [09] and provides a common language to exchange link-layer information of different RANs and MT's battery-level information. It is therefore meant to optimize the $\mathrm{HO}$ process between heterogeneous networks. It uses both the MTs and the networks as information sources. Its most important entity is the Media Independent Handover Function which is an interface between the media specific technology and the MIH users. This entity provides three services to the higher levels [08]:

1. Media Independent Event Service: the MTs subscribe to events such as changing in the state of a link layer, HOs completions, changes in link conditions, etc. These events can be predictive as well: decreasing in RSS can mean that the connection will get lost soon.

2. Media Independent Command Service: MIH users send commands to the Media Independent Handover Function and the Media Independent Handover Function send commands to the access network interfaces. These commands can cause event indications to other MIH users. Thanks to this service, the MIH users can get dynamic information about the situation on the link layer (SNR, bit error rate). Besides, the Media Independent Command Service can be used to subscribe or unsubscribe of certain events, configure thresholds for report events, activate actions on the link layer and even for link layer resource reservation.

3. Media Independent Information Service: it facilitates the exchange of information between MTs and operators on possible HO network candidates.

This information is usually static and it is provided through Information Elements which can be classified in three groups:

a. General information about the available access networks within an area: offered QoS, cost, used frequency bands, maximum data rate, etc.

b. Information concerning to the different PoAs available: channel range, link layer address and geographic location.

c. Access network-, service- or vendor specific IEs. They provide network information about the 
supported higher layer services on the available networks. The IEEE 802.21 has proposed the Media Independent Handover (MIH) services [5] to enhance the handovers across heterogeneous access networks, i.e. vertical handover, and to optimize the service (or session) continuity during handovers, i.e. seamless handover. For this reason, MIH provides generic link layer intelligence and other related network information to upper layers. Particularly, MIH offers a framework of the message flows between handover-related entities to provide information on handover candidate networks and to deliver handover commands. The information service provides a framework and corresponding mechanisms by which a MIH function entity can discover and obtain network information existing within a geographical area to facilitate the handovers. The information service primarily provides a request/response type of mechanism for information transfer. The information may be stored within the MIH layer or maybe presented to some information server from where the MIH layer can access. The information service provides access to static information such as neighboring networks, helping in network discovery. Also, the service may provide access to dynamic information which may optimize link layer connectivity with different networks. This could include link layer parameters such as channel information, MAC addresses of the PoA (Point of Attachment), security information, network type, operator identifier, service identifier, geographical location, etc. It is clear that in current and future environments, dynamic context information from network side entities is very important for the vertical handover decision procedures. Context-aware media independent information server is also proposed for optimized seamless handover procedures [6]. The paper addresses a new concept of a context-aware information server that is able to store, manage and deliver real-time dynamic information retrieved from the network and the terminal side entities, such as the user preferences, running services, mobile nodes.

Rest of the paper is organized as follows: Section 2 present the related work proposed by different researchers. In section 3 , we present the proposed work in which we calculate power consumption, expected lifetime of available networks and describe energy efficient network selection. In Sect 4results are analyzed for energy efficiency. Finally, Conclusions and Future Work are present in Sect 5.

\section{LITERATURE SURVEY}

According to literature survey after studying various IEEE paper, collected some related papers and documents some of the point describe here:

\section{Gamal Abdel Fadeel Mohamed Khalaf et.al (2012)}

In[1], ,A comprehensive approach to vertical handoff in heterogeneous wireless networks" A comprehensive approach to vertical handoff in heterogeneous wireless networksA multi-criteria vertical handoff system sensitive to various mobile-terminals mobility parameters including distance and velocity in a heterogeneous wireless network is analytically formulated and validated via simulations. It is targeted to estimate the essential handoff
Parameters including outage probability, residual capacity, and signal to interference and noise threshold as well as network access cost. In order to avoid the ping pong effect in handoff, a signal evolution prediction system is formulated and its performance is examined. Moreover, the handoff scheme is triggered using an on line handoff initiation-time estimation scheme. When initiated, the handoff procedure begins with a network scoring system based on multi-attribute strategy which results in selection of potentially promising network parameters. Simulation results are shown to track well the analytical formulations.

2. Mohamed A. Al Masri et.al (2015)

In[2]," An Optimized Network Selection and Handover Triggering Scheme for Heterogeneous Self-Organized Wireless Networks the author's proposed derives an analytical expression for calculating the packet loss probability for orthogonal frequency division multiple access based networks. Taking users mobility and spatial distribution into account, this paper develops a Markovian-based framework for evaluating inter-RAT off loading performance. The accuracy of the proposed mobility model and the efficiency of the proposed performance evaluation framework are validated. Results show that MS mobility and vertical handover (VHO) cost significantly affect inter-RAT offloading performance and ignoring their effects could lead to an inaccurate evaluation. When the effects of MS mobility and VHO cost on the performance are ignored, utilizing interRAT offloading decreases the packet loss probability by $90 \%$ and saves energy by 6\%. However, accounting for these effects results in a $32 \%$ increase in the packet loss probability evaluation and a $64 \%$ increase in the energy consumption evaluation.

3. Murad Khan et.al(2014)

In[3], "An Optimized Network Selection and Handover Triggering Scheme for Heterogeneous Self-Organized Wireless Networks". Interference aware vertical handoff decision algorithm for quality of service support in wireless heterogeneous networks they proposed an optimized handover triggering technique. They compare our proposed scheme with existing schemes in context of energy consumption for scanning, frequent and failed handovers, packet loss ratio, and handover delay. The proposed scheme shows superior performance and it outperforms existing schemes used for similar purpose. Moreover, simulation results show the accuracy and performance of the proposed scheme.

4. Silki, Savina Bansal et.al(2013)

IEEE Standard for Local and Metropolitan Area Networks, Media Independent Handover Services, IEEE 802.21 In [10] Five MADM algorithms (SAW, MEW, TOPSIS, AHP and GRA) were considered for network selection by comprising six candidate networks as UMTS, WLAN and WIMAX (two of each kind). Simulation results show that MEW algorithm possesses least handover latency among all the MADM algorithms but network selection is not accurate as desired by user. On the other hand TOPSIS exhibits large handover latency along with desired network selection.

5. C. Guo, Z. Guo, Q. Zhang, and W. Zhu [07]

"A Seamless and Proactive End-to-End Mobility Solution for Roaming Across Heterogeneous Wireless Networks,"

A seamless and proactive scheme for VHOs is proposed. In a VHO scenario, a proactive solution means to access to the network conditions and user's preferences before the VHO decision process. In order to provide such a system, two factors are considered as the most important: network 
condition detection and connection maintenance. The cited solution consists of a novel end-to-end mobility management system with two main parts. The first one is a connection manager used to detect the different network and their conditions. The handoff metric proposed is a QoS-based and includes available bandwidth and access delay and the connection manager accesses to them via WLAN media access control layer sensing. And the second part is a virtual connectivity based on the end-to-end principle and employed to maintain the connection.

6. F. He and F. Wang, [06]

"Position Aware Vertical Handoff Decision Algorithm in Heterogeneous Wireless Networks,"

A first approach to HOs consists of only considering the RSS value. As it was explained in previous sections, this is the traditional approach for HOs which involves changing of PoA when the RSS is below a certain threshold and consequently the current connection is in danger. A classification of these approaches is made in [38]: 1. Relative RSS: if a candidate PoA has better RSS than the current one, then a HO is performed. 2. Relative RSS plus absolute RSS: if a candidate PoA has better RSS than the current one and at the same time the RSS of the current PoA is below a certain threshold, then a HO is performed. 3. Absolute RSS plus hysteresis: if the current RSS is below a certain threshold and the candidate's RSS is higher than the current one plus the hysteresis, then a $\mathrm{HO}$ is performed. 4. Dwell timer: when the decision of performing a $\mathrm{HO}$ has been taken, a dwell timer starts. If after this time, the candidate's conditions remains, then the $\mathrm{HO}$ will be finally made. This timer aims at avoiding the ping-pong effect. However, it also adds a certain HO delay.

\section{PROPOSED WORK}

We have to create more than one wireless network region with number of nodes. The main aim is to achieve QoS, QoE and energy efficiency. First designing bandwidth management algorithm. Then energy management algorithm proposed. Handover is the process of communicating from one region to other region without any interruptions; we apply MIH method with global access point services. Our proposed results should prove the delay is reduced, performance and network lifetime is improved; we show these details via graphical results. All these nodes are indicated as Mobile Users, so they can travel from one region to other without any restrictions. In simulation apart from paper we can show multicasting routing methodology to show our results are best with more destinations as well.

\subsection{Proposed Algorithm}

Inputs: MIH Network Integration,

LLB $=$ Lower Limit of Bandwidth

Output: Successful Handover

Step 1: MN starts a new application

Step 2: Communicates with MIH

Step 3: MIH sends QoS value and recommendation

Step 4: If better network available

Step 5: Initiates Handover to the most suitable

network

else

Step 6: Stays in the current network
2. MN faces network signal issue

Step 1: Communicates with MIH

Step 2: If possible to communicate

Step 3: MIH sends QoS value and recommendation

Step 4: If better network available

Step 5: Initiates Handover to the most suitable network

Else

Step 6: Communicate with BS

Step 7: Gets network information

Step 8: Handovers to the best suitable network

Step 9: End

3. MN faces network performance issue

Step 1: Communicates with MIH

Step 2: MIH sends QoS value and recommendation

Step 3: If better network available

Step 4: Initiates Handover to the most suitable network

Step 05: STOP

\subsection{Proposed Performance Parameters:}

Four performance parameters Throughput, Delay, Network Lifetime and Energy Efficiency are evaluated for MIH Handover. These four parameters are mentioned as follows:

Throughput: Throughput or network throughput is the average rate of successful message delivery over a communication channel. Throughput is measured in bits per second (bit/s or bps [10]).

Throughput $=[($ TransPacketSize * IndiNodeStrength * PackTransSpeed) / Power Consump]

Delay:End-to-End delay refers the time taken for a packet to be transmitted across a network from source to destination. It represents the average data delay for an application or a user experiences when transmitting data. The delay is usually measured in seconds.

Delay $=[($ INodeStrength PTSpeed PConsump $)=$ T imestamp $]$

Timestamp $=$ Timestamp

NetworkLifetime: In order to improve the network lifetime performance of handover in terms of throughput, unnecessary handovers etc.

NetworkLifetime $=[($ TransPacketSize + PackTransSpeed + IndiNodeStrength+NodeMobilityRange $)=($ AverageDelay $)]$

where;

IndiNodeStrength $=$ IndependentNodeStrength

PT Speed $=$ Packet TransmissionSpeed

Pconsump $=$ Power Consumption

Energy Efficiency: In order to improve the energy efficiency of MIH.

Energy Efficiency $=[($ Transission Packet Size * IndiNodeStrength * NodeMobilityRange) / (PackTransSpeed - (AverageDelay + ThersholdTimeStamp)) 


\subsection{Network Parameters for Simulation}

We are using NS3 simulator to perform the practical analysis of designed method. We have designed heterogeneous networks with Wi-Fi,WCDMA,LTE and LTE-A and varying number of mobile stations 20, 40, 60, 80, 100,120.

Table show Network parameters for simulation.

Table 1. Network Parameters for Simulation

\begin{tabular}{|c|c|}
\hline Parameter Name & Value \\
\hline Enter the source node (1-4) & 3 \\
\hline Enter the destination node-1 (13-16) & 14 \\
\hline Enter the destination node-2 (17-20) & 18 \\
\hline Enter the destination node-3 (21-24) & 23 \\
\hline Enter packet size between 1000 to2000 kbps & 1234 \\
\hline Enter your choice between 60 and65 bps & 63 \\
\hline Enter your choice between 100 and 200 & 134 \\
\hline Simulation Time & $25.0 \mathrm{sec}$ \\
\hline Methods & MADM \\
\hline Energy average delay between 5 and 7 & 6 \\
\hline Enter time interval between 5 and 15 & 7 \\
\hline
\end{tabular}

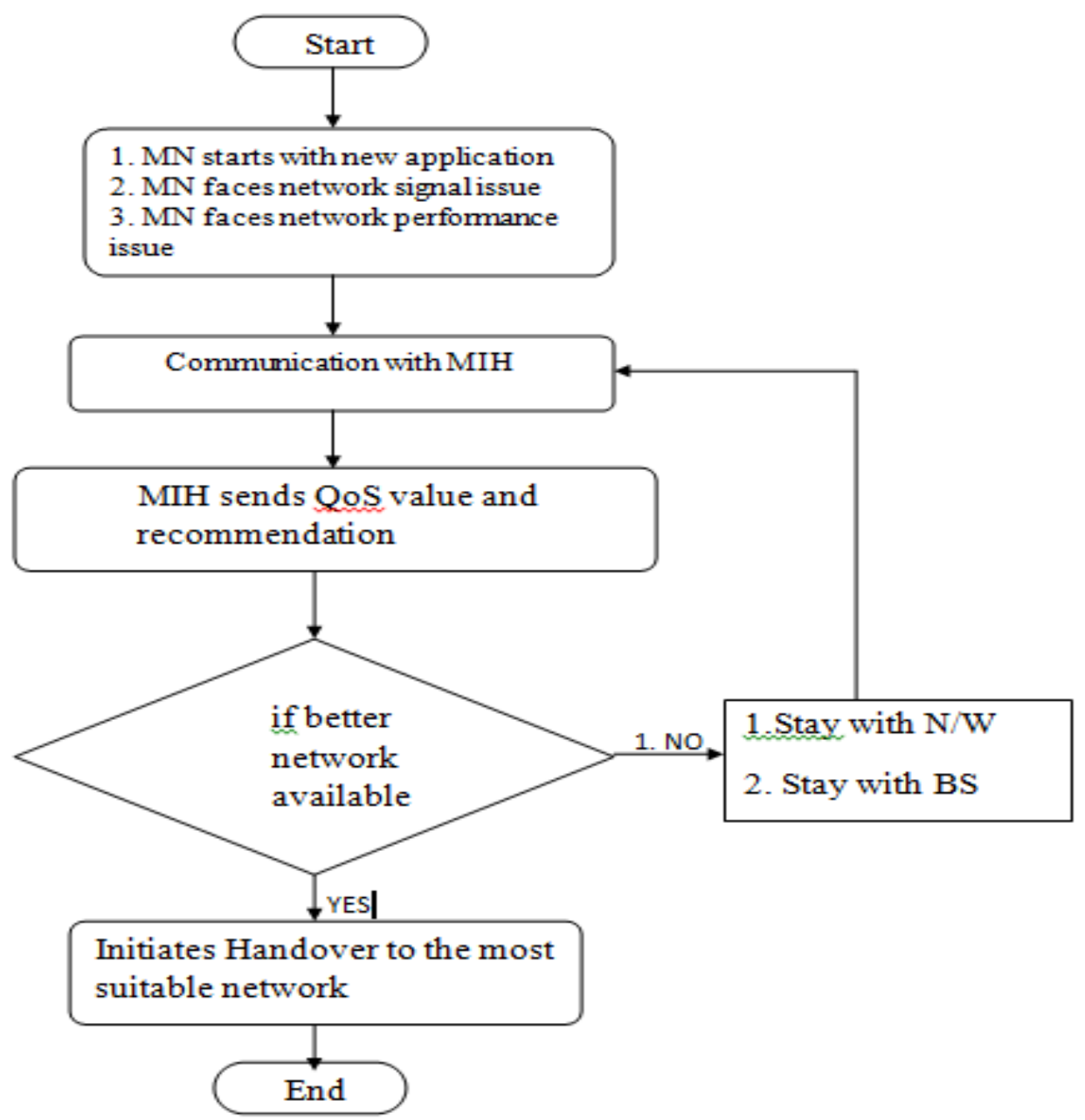

Fig 3.4: Proposed Algorithm 


\section{RESULTS}

Figure 4.1 is showing that proposed method achieving the better performance for Network Lifetime Analysis under different network conditions as compared to existing methods.

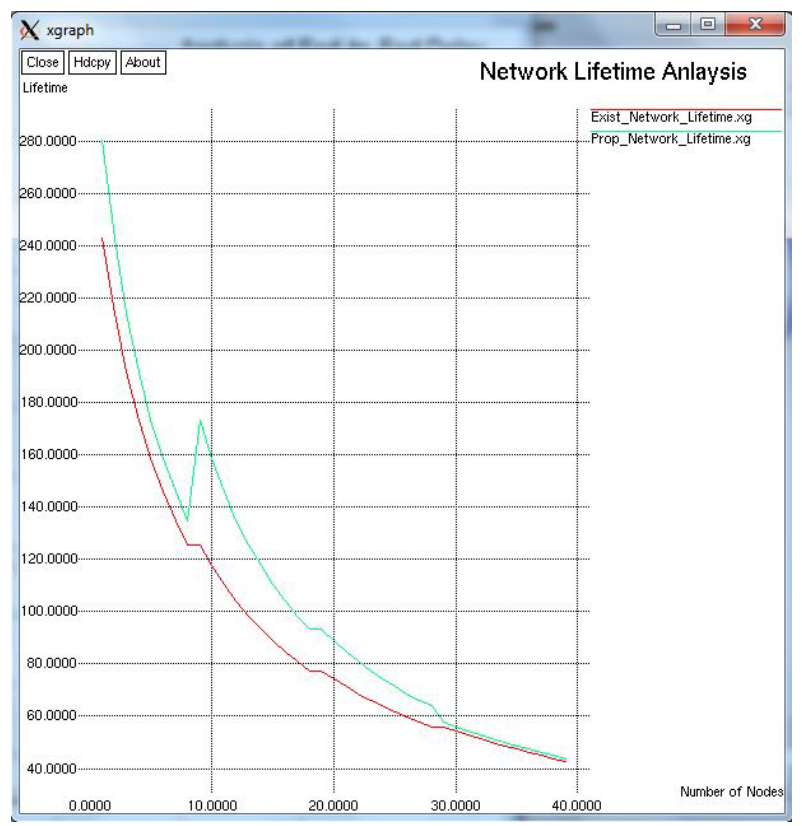

Fig 4.1 Network Lifetime Analysis

Figure 4.3 Is showing that proposed method achieving the better performance for remaining energy efficiency under different network conditions as compared to existing methods.

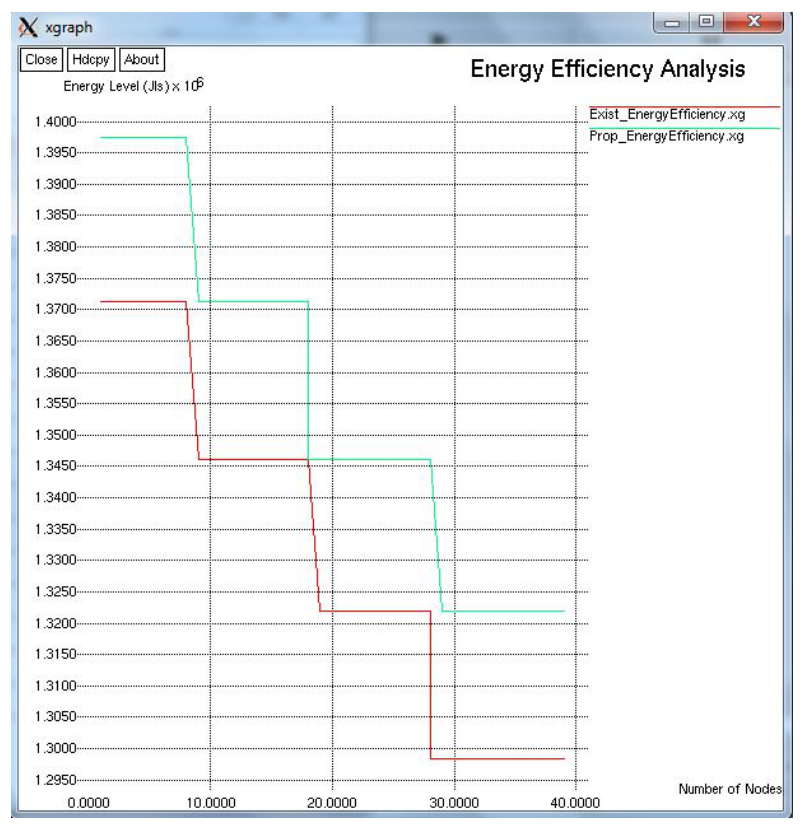

Fig4.3 Energy Efficient Analysis

Figure 4.2 is showing that proposed method achieving the better performance for delay rate under different network conditions as compared to existing methods.

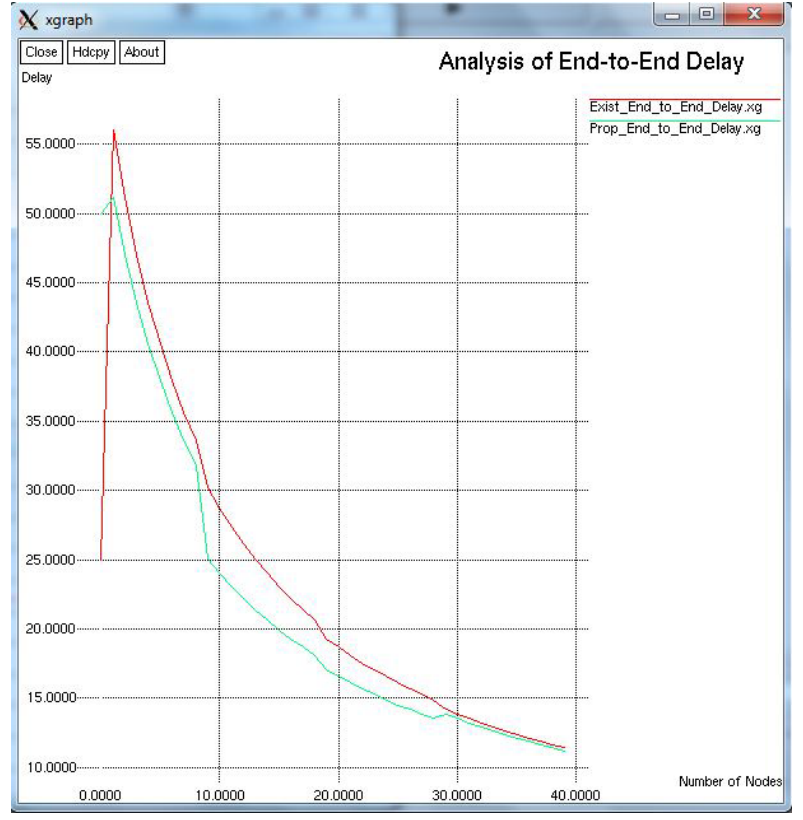

Fig4.2 Analysis of End-to-End Delay

Fig 4.4 is showing that proposed method achieving the average rate of successful message delivery over a communication channel as compared to existing method.

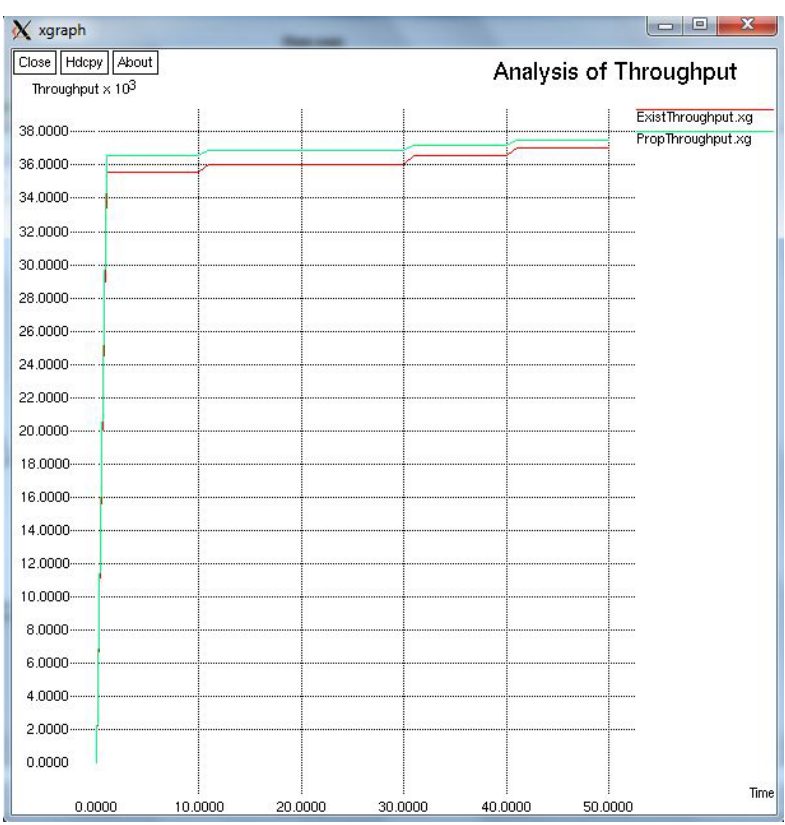

Fig 4.4 Analysis of Throughput

For a section head and a subsection head together (such as Section 3 and subsection 3.1), use no additional space above the subsection head.

\section{CONCLUSION}

We have proposed energy efficient MIH algorithm for heterogeneous network we conclude that in heterogeneous networks, the energy efficiency is increased on the performance measure of throughput, energy, delay and jitter by using this algorithm; we can reduce handover preparation delay and prevent unnecessary handovers. Our propose method gives modified algorithm for vertical handoff scheme considering the parameters required for selection of network 
We have implemented MADM and proposed energy efficient MIH method giving better result. Result shown that, with comparison of MIH, it improves throughput by $35 \%$, Reduce delay rate by $40 \%$ and Increase energy rate by $10 \%$.

\section{REFERENCES}

[1] Gamal Abdel Fadeel Mohamed Khalaf *, Hesham Zarief Badr,"A comprehensive approach to vertical handoff in heterogeneous wireless networks"Electronics, Communications and Computer Department, Faculty of Eng., Helwan, Cairo,Egypt 2012.Brown, L. D., Hua, H., and Gao, C. 2003. A widget framework for augmented interaction in SCAPE.

[2] Murad Khan and Kijun Han,"An Optimized Network Selection and Handover Triggering Scheme for Heterogeneous Self-Organized Wireless Networks"Schoolof Computer Science and Engineering, Kyungpook National University, Republic of Korea 2014.

[3] Celal eken a,*, Serhan Yarkan b, Hseyin Arslan $\mathrm{b}$,"Interference aware vertical handoff decision algorithm for quality of service support in wireless heterogeneous networks" University of Kocaeli, Technical Education Faculty, Electronics and Computer Education Department, 41380 Kocaeli, Turkey 2010.

[4] Dong Ma, Student Member, IEEE, and Maode Ma, Senior Member, IEEE,"A QoS Oriented Vertical Hando_ Scheme for WiMAX/WLAN Overlay Networks" IEEE Transaction on parallel and distributed system vol.23, no.04, APRIL 2012.

[5] Radhwan Mohamed Abdullah *, and Zuriati Ahmad Zukarnain *,"Enhanced Handover Decision Algorithm in Heterogeneous Wireless Network"Received: 25 May 2017; Accepted: 29 June 2017; Published: 14 July 2017.

[6] F. He and F. Wang, "Position Aware Vertical Handoff Decision Algorithm in Heterogeneous Wireless Networks," presented at the 4th International Conference on Wireless Communications, Networking and Mobile Computing, 2008. WiCOM '08, 2008, pp. 1-5.

[7] C. Guo, Z. Guo, Q. Zhang, and W. Zhu, "A Seamless and Proactive End-to-End Mobility Solution for
Roaming Across Heterogeneous Wireless Networks," IEEE Journal on Selected Areas in Communications, vol. 22, no. 5, pp. 834-848, Jun. 2004.

[8] S. Frei, W. Fuhrmann, A. Rinkel, and B. V. Ghita, "Improvements to Inter System Handover in the EPC Environment," in Paris, 2011, pp. 1-5.

[9] K. Taniuchi, Y. Ohba, V. Fajardo, S. Das, M. Tauil, Yuu-Heng Cheng, A. Dutta, D. Baker, M. Yajnik, and D. Famolari, "IEEE 802.21: Media independent handover: Features, applicability, and realization," IEEE Communications Magazine, vol. 47, no. 1, pp. 112-120, Jan. 2009.

[10] IEEE Standard for Local and Metropolitan Area Networks, Media Independent Handover Services, IEEE 802.21-2008,2009

[11] F. Zarai, I. Smaoui, jean-M. Bonnin, and L. Kamoun, "Seamless Mobility in Heterogeneous Wireless Networks," International Journal of Next-Generation Networks, vol. 2, no. 4, pp. 12-31, Dec. 2010.

[12] Energy-Efficient Vertical Handovers José María Rodríguez Castillo jmrc@kth.se 2/25/2013

[13] R. Tawil, G. Pujolle, and O. Salazar, "A Vertical Handoff Decision Scheme in Heterogeneous Wireless Systems," in IEEE Vehicular Technology Conference, 2008. VTC Spring 2008, 2008, pp. $2626-2630$.

[14] “AN INTEGRATED WLAN AND WIMAX ARCHITECTURE WITH QOS-BASED VERTICAL HANDOFF SCHEME “ S. S. SAMBARE1 \& MADHURI R. PAWAR2 1 Pimpri Chinchwad College of Engineering, Pune, Maharashtra, India 2GHRCEM, Wagholi, Pune, Maharashtra, India.

[15] Practical Analysis Vertical Handover Decision (VHD) Algorithm for WIMAX and WLAN S.V. Surwase G.H.Raisoni COEM, Pune S.S. Sambare PCCOE, Pune.

[16] Modified Efficient Handover mechanism For WiFiWiMAX Heterogeneous Network Environment. 1 Prof. S. S. Sambare, 2 Miss. Abhilasha Zatke Department of Computer Engineering PIMPRI CHINCHWAD COLLEGE OF ENGINEERING NIGDI, PUNE-411044. 